\title{
Reducing Length of Stay is not Associated with Increased Readmission Rates Following Lower Extremity Arterial Bypass
}

\author{
Dirichi Arungwa ${ }^{1}$, Andrew Gonzalez² \\ ${ }^{1}$ Indiana University School of Medicine; ${ }^{2}$ Indiana University School of Medicine, Indiana \\ University Health, Department of Vascular Surgery
}

\section{Objective:}

Traditional fee-for-service reimbursement models for medical services have been increasingly replaced with the merit-based and efficiency-driven pay-for-performance initiatives. Financial incentives to decrease expenditures have added pressure on hospitals and providers to reduce length of stay (LOS). Concern has been raised that this strategy may unwittingly increase readmission rates. We sought to better understand potential trade-offs between these two outcome measures for hospitals performing lower extremity arterial bypass (LEAB).

\section{Methods:}

We performed a retrospective observational cohort study of 479,366 Medicare beneficiaries undergoing LEAB in 1,708 hospitals from 2005-2009. Using LOS quintiles and a multiple logistic regression model, we generated risk-adjusted readmission rates (RARR) accounting for age, gender, pre-existing comorbidities, non-elective admission status, post-operative complications, and discharge destination. We then compared RARRs across LOS quintiles. Finally, we evaluated the association between hospital LOS on readmissions performance after adjusting for hospital volume, location, teaching status, bed size, and non-home discharges.

\section{Results:}

The mean hospital 30-day RARR was 14.5\% (SD 3.5\%) and the median LOS 4 days (range 3-6 days). Hospitals with the shortest median LOS (2.3 days) had slightly lower readmission rates compared to those with the highest median LOS (9.2 days) $(13.3 \%$ vs $15.8 \%, p<0.001)$. The Spearman correlation coefficient was $0.15(p<0.001)$, indicating a very weak correlation between LOS and RARR.

\section{Conclusion and Potential Implications:}

At the hospital-level, there appears to be little tradeoff between LOS and RARR, implying these outcomes measure different domains of hospital quality and can be used as indicators of care quality and the likelihood for post-discharge adverse events. As it is possible for hospitals to perform well on both measures, pay-for-performance initiatives may consider incentivizing reducing LOS and readmissions. 